Aim of the study: This retrospective study attempts to evaluate the influence of serum vascular endothelial growth factor C (VEGF-C), microvessel density (MVD) and lymphatic vessel density (LMVD) on the result of tumour treatment in women with cervical cancer. Material and methods: The research was carried out in a group of 58 patients scheduled for brachytherapy for cervical cancer. All women were patients of the Department and University Hospital of Oncology and Brachytherapy, Collegium Medicum in Bydgoszcz of Nicolaus Copernicus University in Toruń. VEGF-C was determined by means of a quantitative sandwich enzyme immunoassay using a human antibody VEGF-C ELISA produced by Bender MedSystem, enzyme-linked immunosorbent detecting the activity of human VEGF-C in body fluids. The measure for the intensity of angiogenesis and lymphangiogenesis in immunohistochemical reactions is the number of blood vessels within the tumour. Statistical analysis was done using Statistica 6.0 software (StatSoft, Inc. 2001). The Cox proportional hazards model was used for univariate and multivariate analyses. Univariate analysis of overall survival was performed as outlined by Kaplan and Meier. In all statistical analyses $p<0.05$ (marked red) was taken as significant.

Results: In 51 patients who showed up for follow-up examination, the influence of the factors of angiogenesis, lymphangiogenesis, patients' age and the level of haemoglobin at the end of treatment were assessed. Selected variables, such as patients' age, lymph vessel density (LMVD), microvessel density (MVD) and the level of haemoglobin $\mathrm{Hb}$ ) before treatment were analysed by means of Cox logical regression as potential prognostic factors for lymph node invasion. The observed differences were statistically significant for haemoglobin level before treatment and the platelet number after treatment. The study revealed the following prognostic factors: lymph node status, FIGO stage, and kind of treatment. No statistically significant influence of angiogenic and lymphangiogenic factors on the prognosis was found.

Conclusion: Angiogenic and lymphangiogenic factors have no value in predicting response to radiotherapy in cervical cancer patients.

Key words: cervical cancer, angiogenesis, lymphangiogenesis, VEGF-C.

\section{Angiogenesis and lymphangiogenesis as prognostic factors after therapy in patients with cervical cancer}

\author{
Marta Biedka ${ }^{12}$, Roman Makarewicz' ${ }^{1}$ Ewa Kopczyńska ${ }^{3}$, \\ Andrzej Marszałek ${ }^{4,5}$, Alina Goralewska ${ }^{6}$, Hanna Kardymowicz ${ }^{6}$
}
${ }^{1}$ Chair and Clinic of Oncology and Brachytherapy, Ludwik Rydygier Collegium Medicum, Bydgoszcz, Nicolaus Copernicus University, Toruń, Poland
${ }^{2}$ Radiotherapy Department I, Oncology Centre, Bydgoszcz, Poland
${ }^{3}$ Chair and Department of Pathobiochemistry and Chemistry, Ludwik Rydygier Collegium Medicum in Bydgoszcz, Nicolaus Copernicus University, Toruń, Poland
${ }^{4}$ Chair and Department of Clinical Pathomorphology, Ludwik Rydygier
Collegium Medicum, Bydgoszcz, Nicolaus Copernicus University, Toruń, Poland
${ }^{5}$ Chair and Department of Clinical Pathomorphology, Poznan University of Medical
Sciences, Poland
${ }^{6}$ Diagnostic Ward, Oncology Centre, Bydgoszcz, Poland

\section{Introduction}

Poland is among European countries with the highest levels of cervical cancer, with as many as 3345 new incidents and 1819 deaths in 2006 alone [1]. It affects young women, and the incident rate increases with age, the highest risk age group being 45-55 years. The basic methods of treatment are surgery, radiation and chemotherapy. All of these are applied in different combinations. The choice of treatment is mainly based on clinical evaluation of tumour stage according to the International Federation of Gynecology and Obstetrics (FIGO staging system).

The choice of treatment is dependent on prognostic factors such as tumour size, patient's age and general condition, type and grade score of tumour differentiation (numerical: G1 up to G4), invasion of lymph vessels or blood vessels, lymph node status and the level of haemoglobin before treatment. But most authors still search for a new predictive factor which would help determine the patients with high risk of metastasis to lymph nodes and/or relapse [1-10].

Angiogenesis is the process of formation of a new blood vessel from the existing vascular network. It is essential for tumour growth, progression and metastasis. It plays a crucial role in many phenomena, both physiological and pathological, and it consists of many stages [11, 12].

Numerous data indicate that the induction of angiogenesis can proceed in two ways: either through mutations activating oncogenes or mutations inhibiting the functions of tumour suppressor genes [11]. In the case of larger tumours, blood supply is compromised. It is believed that cellular hypoxia is a major, though not the only, factor initiating the mechanism of angiogenesis. Low oxygen concentration induces the synthesis of larger amounts of transcription factors, including HIF (hypoxia-inducible factor), in the cell [13]. It is associated with an increase in the expression and activity of the gene encoding vascular endothelial growth factor (VEGF), which is considered the strongest and a key promoter of angiogenesis [14].

The lymphatic system is the most common way of spreading cancer cells, starting in the very early stages of the disease. Lymphatic capillaries can sprout from pre-existing lymphatic vessels, in the same way as blood vessels. There is a hypothesis that the new vessels are formed in the process called lymphangiogenesis. Until recently it was assumed that the lymph system is "anatomically" absent in the tumour and that the formation of lymphatic ves- 
sels is secondary to the process of angiogenesis, although simultaneous growth of blood and lymph vessels is also possible [15]. Currently, more and more facts speak in favour of the assumption that the process of lymphangiogenesis is present from the very beginning of the development of cancer and coexists with the process of angiogenesis, resulting from, among other things, stimulation of VEGF-C, which enhances the process of angiogenesis through VEGFR-2 receptor and/or stimulates the process of lymphangiogenesis through VEGFR-3 receptor [16].

\section{Material and methods}

The research was carried out in a group of 58 patients scheduled for brachytherapy for cervical cancer. All women were patients of the Department and University Hospital of Oncology and Brachytherapy, Collegium Medicum in Bydgoszcz of Nicolaus Copernicus University in Toruń, between 2005 and 2006. In these patients, an analysis of survival was conducted 5 years after treatment.

Each patient was treated in accordance with the standard procedures for cervical cancer treatment. In stage 1-2A patients preoperative brachytherapy was applied. Two methods of brachytherapy were used: HDR (high dose rate), where 30 Gy were administered in 4 fractions, and LDR (low dose rate), where the rate of dose delivery is 45-50 Gy in 2 fractions. Five weeks after the radiation therapy, patients underwent wide hysterectomy with or without adnexa and with bilateral removal of the lymph nodes of the true pelvis. Adjuvant radiotherapy or chemotherapy was necessary in case of metastasis to lymph nodes, deep infiltration, infiltration of lymphovascular spaces, grade score G2, G3. In patients with indications for chemotherapy, 4-5 cycles of cisplatin, dosed $50-100 \mathrm{mg} / \mathrm{m}^{2}$, were administered intravenously every 7 days. Teletherapy consisted of radiation to the pelvis in a total dose of 44-50.4 Gy, in a fraction dose of 1.8-2.0 Gy. In patients in stages $2 \mathrm{~B}-4$ and those disqualified from surgical treatment, radiotherapy (brachytherapy + teletherapy) or radiochemotherapy (teletherapy with chemotherapy + brachytherapy at the same time) was applied as basic treatment. In patients qualified for brachytherapy alone, it was administered by means of HDR, at the rate of dose delivery of 36 Gy in 6 fractions. $2 \mathrm{ml}$ of blood was collected from the basilic vein on an empty stomach, once, at seven o'clock in the morning, before the start of the treatment. Tumour samples from the cervix uteri affected by cancer were taken during the first brachytherapy under a short general anaesthesia.

VEGF-C was determined by means of a quantitative sandwich enzyme immunoassay ELISA using a human antibody VEGF-C ELISA produced by Bender MedSystem, enzyme-linked immunosorbent detecting the activity of human VEGF-C in body fluids. The measure for the intensity of angiogenesis and lymphangiogenesis in immunohistochemical reactions is the number of visible blood vessels within the tumour. The staining is based on the reaction of the antigen localised on vessel intraepithelial cells, with the appropriate monoclonal antibody (human Podoplanin. Mouse monoclonal antibody; clone 4D5aE5E6BMS 1105 Bender Medsystem) and (human CD-31 DAKO-CD31, code M0823).

\section{Methods of statistical analysis}

Statistical analysis was performed using the computer software Statistica 6.0 (StatSoft, Inc. 2001). The influence of selected parameters on progression-free survival was assessed by the Cox proportional hazards model. The variables which in Cox regression analysis gained statistical significance were included in the initial model. Analysis of survival in each group of patients was conducted by means of the KaplanMeier method. In all statistical analyses $p<0.05$ (marked red) was taken as significant.

\section{Results}

The research was carried out in a group of 58 cervical cancer patients. Detailed characteristics are given in Table $1 a, b$. 51 out of 58 analysed patients came back for a follow-up visit and in the remaining 7 women the course of the disease was unknown, so they were excluded from the analysis of

Table 1a. General characteristics of the group of cervical cancer patients

\begin{tabular}{|lcccc} 
& Before treatment & \multicolumn{3}{c}{ 6 weeks after treatment } \\
\cline { 2 - 4 } & & CR & PR & PD \\
\hline FIGO stage & $N=58(\%)$ & $N=37(\%)$ & $N=4(\%)$ & $N=10(\%)$ \\
\hline Stage I & $23(39.7)$ & $18(78.2)$ & $0(0)$ & $1(4.3)$ \\
Stage II & $18(31)$ & $13(72.2)$ & $1(5.5)$ & $3(16.6)$ \\
Stage III & $13(22.4)$ & $6(46.1)$ & $3(23)$ & $3(23)$ \\
Stage IV & $4(6.9)$ & $0(0)$ & $0(0)$ & $3(75)$ \\
\hline Grade score of tumour differentiation & $N=31(\%)$ & $N=19(\%)$ & $N=2(\%)$ & $N=5(\%)$ \\
G1 & $2(6)$ & $2(100)$ & $0(0)$ & $0(0)$ \\
G2 & $21(68)$ & $13(61.9)$ & $2(9.5)$ & $4(19)$ \\
G3 & $8(26)$ & $4(50)$ & $0(0)$ & $1(12.5)$ \\
\hline Kind of planning treatment & $N=58(\%)$ & $N=36(\%)$ & $N=4(\%)$ & $N=11(\%)$ \\
Brachytherapy + surgery & $33(56.8)$ & $24(72.7)$ & $0(0)$ & $3(9)$ \\
Brachytherapy + teletherapy & $17(29.3)$ & $9(52.9)$ & $3(17.6)$ & $5(29.4)$ \\
Brachytherapy & $8(13.7)$ & $3(37.5)$ & $1(12.5)$ & $3(37.5)$
\end{tabular}

$C R$ - complete response; $P R$ - partial response; $P D$ - progression of disease 
Table $1 \mathrm{~b}$. Lymph node status in operated patients

\begin{tabular}{|c|c|c|c|c|}
\hline \multicolumn{5}{|c|}{ Patients' characteristics } \\
\hline & \multirow[t]{2}{*}{ Before treatment } & \multicolumn{3}{|c|}{6 weeks after treatment } \\
\hline & & CR & PR & PD \\
\hline & $N=33(\%)$ & $N=23(\%)$ & $N=0(\%)$ & $N=3(\%)$ \\
\hline NO - 19 & $19(57.7)$ & $13(68.4)$ & $0(0)$ & $0(0)$ \\
\hline$N(+)-12$ & $12(36.3)$ & $9(75)$ & $0(0)$ & $3(25)$ \\
\hline$N x-2$ & $2(6.1)$ & $1(50)$ & $0(0)$ & $0(0)$ \\
\hline
\end{tabular}

NO without the involvement of lymph nodes

$N(+)$ metastasis to the lymph system

Nx unknown status of the lymph nodes

the response to treatment. The total follow-up observation period of the 58 patients ranged between 2 and 31 months (mean follow-up observation period was 9.7 months). Out of 51 women 20 (39.2\%) had a relapse of the cancer, 16 (80\%) had a local relapse, and 4 (20\%) had metastases, including 2 with another cancer diagnosed. The mean time of relapse was 8.2 months (ranging between 1 and 23 months). Detailed data concerning age, VEGF-C concentration in blood serum, microvessel density (MVD), and lymphatic vessel density (LMVD) are presented in Table 2.

Fifty-one patients were analysed for the impact of selected parameters on survival without disease progression assessed by univariate analysis of Cox proportional hazards regression. The analysis showed a statistically significant influence of haemoglobin concentration before treatment $(p=0.008)$, and platelet count after treatment $(p=0.000170)$ on survival. The other clinicopathological parameters such as age, concentration of VEGF-C serum, lymph vessel density in the tumour (LMVD), and microvessel density in the tumour (MVD) had no impact on survival of patients with cervical cancer (Table 3).

In order to select the independent prognostic factors a multivariate Cox backward stepwise analysis was conducted. For the initial model we included factors whose impact on time to progression in univariate analysis was statistically significant, and it was found that the only independent prognostic factor was the number of platelets (PLT) after treatment.
In the next stage of the study the survival probability for each group was estimated by means of the Kaplan-Meier method. The study confirmed that lymph node status has a significant impact on survival: in women with lymphatic metastasis, survival time was shorter compared to women free of metastases (test statistic $-2.88695, p=0.00389$ ) (Fig. 1). According to research by other authors, FIGO stage has an impact on survival; indeed, FIGO I-II patients lived significantly longer compared to those with advanced disease, and the result was statistically significant (test statistic 2.03536, $p=0.04181$ ) (Fig. 2). On analysing the probability of survival in patients with the degree of tumour differentiation G1-G2 vs. G3, the differences observed between the groups did not reach statistical significance (test statistic $0.143718, p=0.88572$ ), as was the case with teletherapy, which was not shown to affect survival (test statistic $0.5869814, p=0.55722$ ). The study confirmed a statistically significant effect of genital tract surgery on survival. Women who had surgery performed had longer survival time (test statistic 2.371850, $p=0.01770$ ) (Fig. 3). Unfortunately, despite studies by other authors indicating a relationship between angiogenic and lymphangiogenic parameters, we did not manage to demonstrate an impact of VEGF-C concentration in serum (test statistic 0.2309617, $p=0.81734$ ) and microvessel density (LMVD) (test statistic -0.036349 , $p=0.97100$ ) on the survival of women with cervical cancer (Figs. 4, 5).

Table 2. Results of univariate Cox proportional hazards model in patients with cervical cancer

\begin{tabular}{|c|c|c|c|c|c|c|}
\hline & $N=$ death + live & death & live & Index $\beta$ & $\beta$ & $p$ \\
\hline Age & 53 & 26 & 27 & 0.0082 & 1.0083 & 0.5756 \\
\hline Serum VEGF-C & 42 & 22 & 20 & 0.000444 & 1.0004 & 0.7442 \\
\hline Lymphatic vessel density LMVD & 31 & 17 & 14 & -0.033726 & 0,966836 & 0.364187 \\
\hline Leucocyte WBC & 52 & 25 & 27 & 0.091573 & 1.095896 & 0.227157 \\
\hline Haemoglobin before treatment $\mathrm{Hb}$ & 52 & 25 & 27 & -0.263270 & 0.768534 & 0.008832 \\
\hline Haemoglobin after treatment $\mathrm{Hb}$ & 53 & 26 & 27 & -0.211829 & 0.809103 & 0.057166 \\
\hline Platelet count PLT & 53 & 26 & 27 & 0.002308 & 1.002311 & 0.187118 \\
\hline Platelet count after treatment PLT & 51 & 24 & 27 & 0.004488 & 1.004498 & 0.000170 \\
\hline Microvessel density MVD & 13 & 7 & 6 & 0.030219 & 1.030680 & 0.631832 \\
\hline
\end{tabular}

In statistical analyses $p<0.05$ (marked red) was taken as significant. 


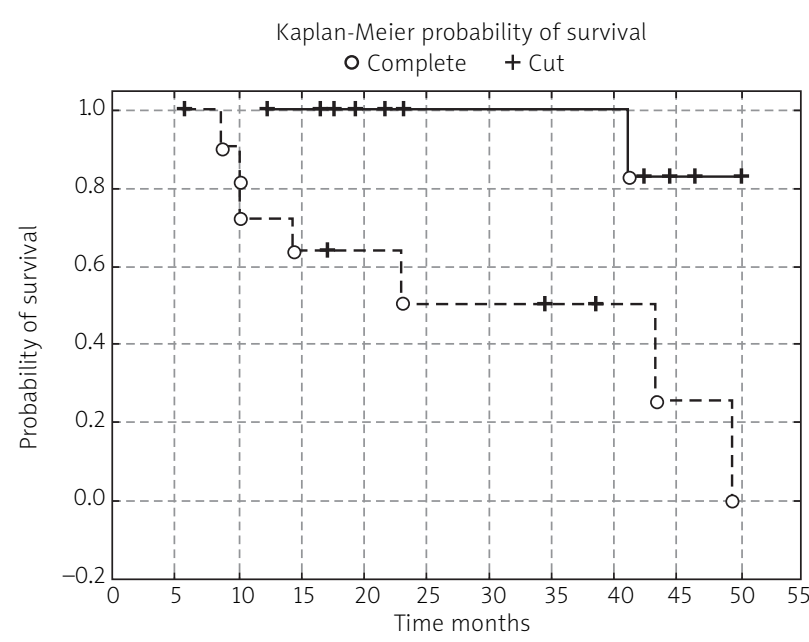

$-\mathrm{N}(-) \quad---\mathrm{N}(+)$

Fig. 1. Estimated overall survival probablities in group of patients whithout lymph node metastasis vs. women with lymph node metastasis

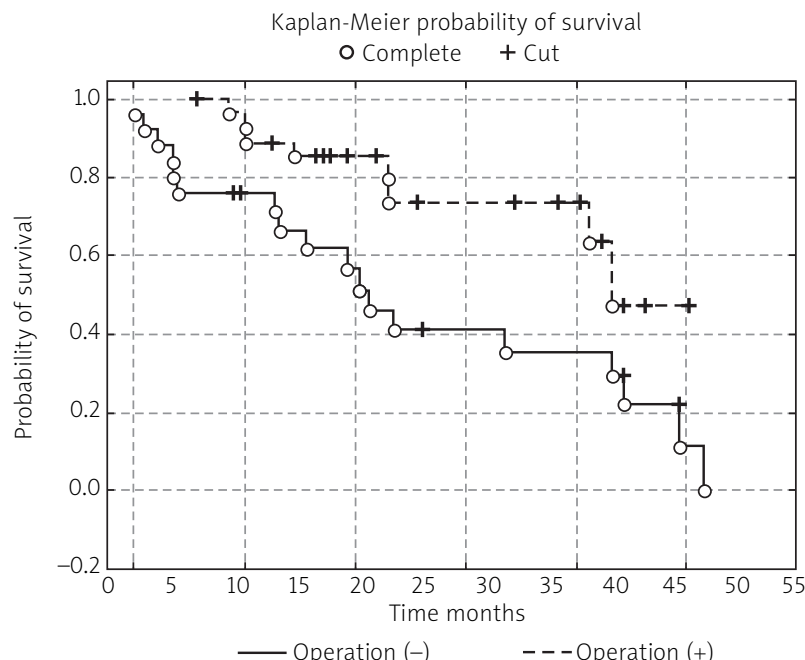

Fig. 3. Estimated overall survival probablities in group of patients with operation vs. women without operation

\section{Discussion}

Many authors have suggested that higher lymphatic vessel density (LMVD) and overexpression of VEGF-C in tumour correlates with metastasis to the lymph nodes and bad prognosis [21-23]. Such a relationship is confirmed in oesophageal, stomach, thyroid and pancreatic cancer. It makes it possible to assume that the concentration of VEGF-C circulating in the organism should be higher, although the results obtained by different authors are different [13, 17-21, 24].

Many data from the published literature indicate that lymphatic vessel density (LMVD) in tumours may correlate with overall survival and disease-free survival rate. It is assumed that intensification of lymphangiogenic processes may lead to tumour progression and worse prognosis. The correlation between lymphatic vessel density (LMVD) and worse prognosis was confirmed in breast cancer, in melanoma and in head and neck cancers, but it was not confirmed in oral and ovarian cancer [13, 17-21]. In the study by Broll et al. [25] the

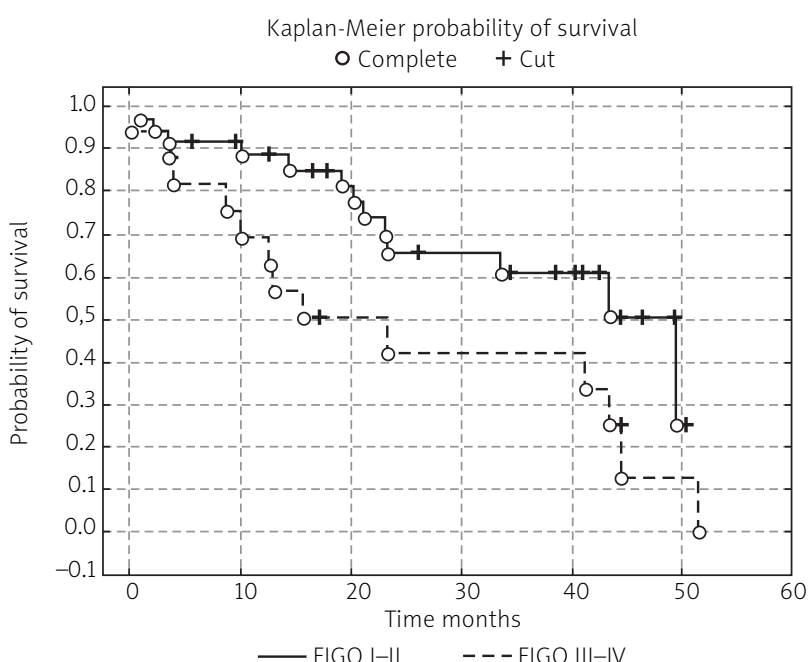

Fig. 2. Estimated overall survival probablities in group of patients FIGO stage I-II vs. patients with advanced disease

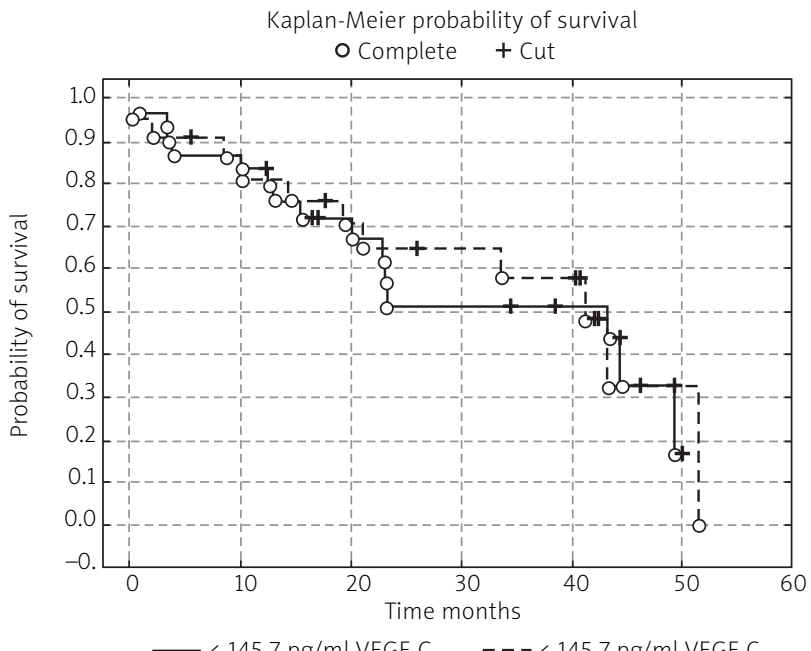

Fig. 4. Estimated overall survival probablities in group of patients $<145.7 \mathrm{pg} / \mathrm{ml}$ VEGF-C in serum vs. women with $>145.7 \mathrm{pg} / \mathrm{ml}$ VEGF-C in serum

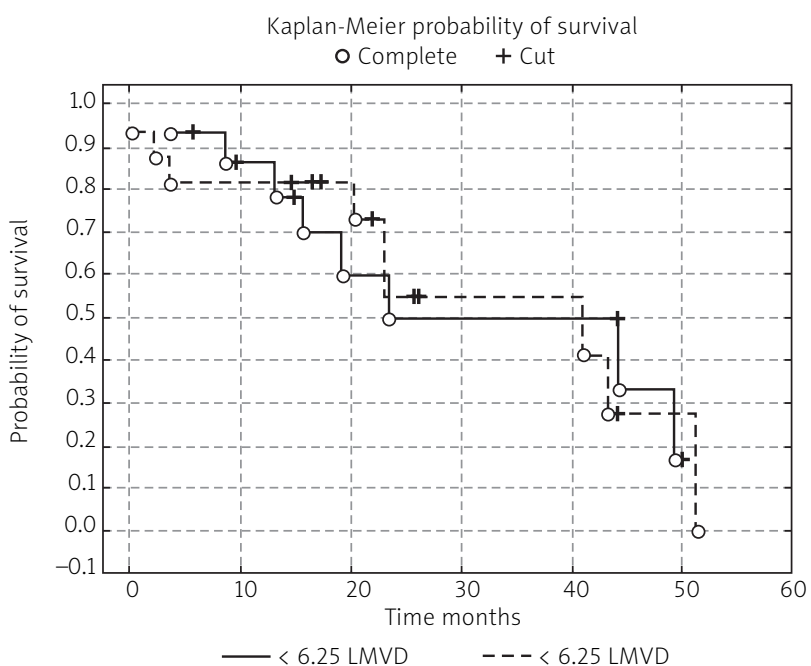

Fig. 5. Estimated overall survival probalities in group of patients with $<6.25$ lymphatic vessel density (LMVD) vs. women with $>6.25$ lymphatic vessel density (LMVD) 
influence of serum VEGF-C concentration on overall survival in colorectal cancer was confirmed and the result was statistically significant. Obermair et al. [2] analysed 166 cervical cancer patients in FIGO stage 1B. The investigation included 102 women with microvessel density $<20$ in the field of view vs. 64 patients with microvessel density $>20$ in the field of view. The author estimated 5-year survival rate as $89,7 \%$ and $63 \%$ respectively. Gasińska et al. [26] obtained similar results. She investigated cervical uteri patients and demonstrated that microvessel density (MVD) in tumour is a significant prognostic factor, and the obtained result reached the level of statistical significance. Li et al. [27] analysed 147 colon cancer patients and transforming growth factor $\beta 1$ (TGF- $\beta 1$ )-Smad and vascular endothelial growth factor C (VEGF-C)/VEGF receptors for exploring changes in pathway members and their influence on lymphangiogenesis and clinicopathological features. Lymphatic vessel density in colon cancer tissues was significantly higher than in normal colonic tissues. VEGF-C expression positively correlated with lymphatic vessel count. Analysis using the Kaplan-Meier method indicated that patients with VEGF-C-positive tumours had significantly shorter overall survival and tumour-free survival time than those with VEGF-C-negative tumours. Similar results were obtained by Osinsky et al. [28] in one 105 resected gastric cancer specimens. In other studies no such relationship was confirmed, for instance Fujimoto et al. [29] in his study on pancreatic cancer did not confirm that microvessel density (MVD) in tumour is of prognostic significance. The interpretation of the present study poses some difficulties. The small number of patients in groups with complete response (CR), partial response (PR) and progression of the disease (PD) was the probable reason for no influence of pro-angiogenic and lymphangiogenic factors on the response to treatment, although other reasons also cannot be excluded. In the published literature an explanation of this situation has been sought. It is emphasised that the key role is played by the ignorance of different mechanisms at a molecular level, the small number of patients taking part in studies, and, mostly, no established methodological standards influencing the results of the conducted tests [30-32].

Many authors have demonstrated that haemoglobin concentration is a prognostic factor in cervical cancer, which has also been confirmed in our study, where in univariate Cox analysis the pretreatment haemoglobin level was a statistically significant factor $(r=-0.263270, p=0.008832)$. As for haemoglobin concentration after treatment, a trend has also been observed, but it did not reach statistical significance $(r=-0.0211829 ; p=0.057166)[5,9,10]$.

In the published sources there have been few authors investigating the relationship between the level of haemoglobin and the processes of angiogenesis and lymphangiogenesis. Ferrero et al. [9] analysed 72 ovarian cancer patients. He demonstrated an inverse correlation between the level of haemoglobin and microvessel density (MVD) in tumour. Such a relationship was not confirmed by other authors dealing with this subject, such as Gasińska or Kay Akin [26, 33, 34].

Low haemoglobin level affects the increase in hypoxia in tumour. It is common knowledge that hypoxia is the strongest promoter of the processes of angiogenesis, and owing to it we observe an increase in the produced pro-angiogenic factors. Therefore, the level of haemoglobin should correlate with the processes of new vessel formation. The lack of such observations may result from the fact that this process is more complex and probably dependent on a multitude of parameters. High vessel density in tumour allows for better nourishment and quicker growth of cancerous cells. On the other hand, it has an influence on better penetration of chemotherapeutic agents inside the tumour and greater efficiency of radiotherapy [26, 33, 34].

Dunst [33] analysed the level of haemoglobin before treatment in 87 cervical uteri patients. He demonstrated that after 3 years the percentage of local control was $85 \%$ with the values of haemoglobin above $13 \mathrm{~g} / \mathrm{dl}$, and it dropped to $60 \%$ with the values of haemoglobin below $11 \mathrm{~g} / \mathrm{dl}$. Haemoglobin concentration had a crucial influence on overall survival rate: $79 \%$ vs. $32 \%$. The author found that haemoglobin concentration below $11 \mathrm{~g} / \mathrm{dl}$ before treatment is the strongest prognostic factor.

Cancer can occur with thrombocythaemia, which is connected with cancer stage, as it can quite frequently be observed in women with cervical cancer [35-37]. Thus, the relationship between platelet counts before treatment and after treatment may correlate with the processes of angiogenesis and lymphangiogenesis. In our study it has been demonstrated that the number of platelets after treatment was an independent prognostic factor.

Haensgen et al. [35] studied 41 patients with cervical cancer in FIGO stage IB-IVA, qualified for primary radiotherapy. The author demonstrated a relationship between VEGF serum levels and platelet count $(p<0.01)$. In women with a response to treatment, platelet count was lower compared to the group with disease progression.

Advances in the understanding of molecular biology have initiated a lot of scientific research. Yet, it still remains a challenge to translate the information that has been acquired into the clinical data in order to bring tangible benefits in daily work with patients. Research tools and the new technologies that have been described in this study can certainly bring us closer to the goal.

\section{Conclusions}

On the basis of the performed study and the analysis of the results the following conclusions were drawn: Angiogenic and pro-angiogenic factors have no value in predicting response to radiotherapy in cervical cancer patients. Hopefully, carrying out further research on a larger number of patients will enable us to understand the mechanisms of angiogenesis and lymphangiogenesis better.

\section{References}

1. Didkowska J. Nowotwory szyjki macicy w Polsce - epidemiologiczny bilans otwarcia i perspektywy. Ginekol Pol 2006; 8: 660-6.

2. Obermair A, Wanner C, Bilgi S, Speiser P, Kaider A, Reinthaller A, Leodolter S, Gitsch G. Tumor angiogenesis in stage IB cervical cancer: correlation of microvessel density with survival. Am J Obstet Gynecol 1998; 178: 314-9.

3. Horn L, Hentschel B, Galle D, Bilek K. Extracapsular extension of pelvic lymph node metastases is of prognostic value in carcinoma of the cervix uteri. Gynecol Oncol 2008; 108: 63-7. 
4. Lee J, Kim H, Jung J, Lee M, Park C. Expression of vascular endothelia growth factor in adenocarcinomas of the uterine cervix and its relation to angiogenesis and p53 and c-erbB-2 protein expression. Gynecol Oncol 2002: 85: 469-75.

5. Fuso L, Mazzola S, Marocco F. Pretreatment serum hemoglobin level as a predictive factor of response to neoadjuvant chemotherapy in patients with locally advanced squamous cervical carcinoma: A preliminary report. Gynecol Oncol 2005; 99: 187-91.

6. Kodama J, Seki N, Ojima Y, Nakamura K, Hongo A, Hiramatsu Y. Prognostic factors in node-positive patients with stage IB-IIB cervical cancer treated by radical hysterectomy and pelvic lymphadenectomy. Int J Gynaecol Obst 2006; 93: 130-5.

7. Sakuragi N. Up-to-date management of lymph node metastasis and the role of tailored lymphadenectomy in cervical cancer. Int J Clin Oncol 2007; 12: 165-75.

8. Horn L, Fischer U, Raptis G, Bilek K, Hentschel B. Tumor size is of prognostic value in surgically treated FIGO stage II cervical cancer. Gynecol Oncol 2007; 107: 310-5.

9. Ferrero A, Zola P, Mazzola S. Pretreatment serum hemoglobin level and a preliminary investigation of intratumoral microvessel density in advanced ovarian cancer. Gynecol Oncol 2004; 95: 323-9.

10. Hefler L, Mayerhofer K, Leibman B, Obermair A, Reinthaller A Kainz C, Tempfer C. Tumor anemia and thrombocytosis in patients with vulva cancer. Tumour Biol 2002; 21: 309-314.

11. Conway E, Collen D, Carmeliet P. Molecular mechanism of blood ves sel growth. Cardiovasc Res 2001; 49: 507-21.

12. Drake C, La Rue A, Ferrara N. VEGF regulates cell behavior during vas culogenesis. Dev Biol 2000; 224: 178-88

13. Schoppmann S, Fenzl A, Schindl M, et al. Hypoxia inducible factor-1 $\alpha$ correlates with VEGF-C expression and lymphangiogenesis in breast cancer. Breast Cancer Res Treat 2006; 99: 135-14.

14. Watanabe Y, Dvorak H. Vascular permeability factor/vascular endothelial growth factor inhibits anchorage-disruption-induced apop tosis in microvessel endothelial cells by inducing scaffold formation. Exp Cell Res 1997; 233: 340-9.

15. Trappen P, Peper M. Lymphangiogenesis in human gynecological cancer. Angiogenesis 2005; 8: 137-45.

16. Alitalo K, Carmeliet P. Molecular mechanisms of lymphangiogenesis in health and disease. Cancer Cells 2002; 4: 219-26.

17. Gao P, Zhou GY, Yin G, Liu Y, Liu ZY, Zhang J, Hao CY. Lymphatic vessel density as a prognostic indicator for patients with stage I cervical carcinoma. Hum Pathol 2006; 37: 719-25.

18. Thiele T, Sleeman J. Tumor-induced lymphangiogenesis: a target for cancer therapy? J Biotechnol 2006; 124: 224-41.

19. Valencaka J, Heere-Ressa E, Koppa T, Schoppmann S, Kittlera H, Pehambergera $\mathrm{H}$. Selective immunohistochemical staining shows significant prognostic influence of lymphatic and blood vessels in $\mathrm{pa}$ tients with malignant melanoma. Eur J Cancer 2004; 40: 358-64.

20. Gao Y, Zhong W, Mu D, et al. Distributions of angiogenesis and lymphangiogenesis in gastrointestinal intramucosal tumors. Ann Surg Oncol 2008; 15: 1117-23.

21. Van Trappen P, Pepper M. Lymphangiogenesis and Lymph node microdissemination. Gynecol Oncol 2001; 82: 1-3.

22. Gisterek I, Sedlaczek P, Kornafel J, et al. Serum vascular endothelial growth factor in patients with pharyngeal and laryngeal squamous cell carcinoma treated with radiotherapy. Am J Otolaryng 2007; 28: 73-7.

24. Biedka M, Makarewicz R, Lebioda A, Kardymowicz H, Goralews ka A. Naczyniowo-śródbłonkowy czynnik wzrostu C jako czynnik predykcyjny w raku szyjki macicy. Wspolczesna Onkol 2010; 14 87-92.

25. Broll R, Erdmann H, Duchrow M, Oevermann E, Schwandner O, Markert U, Bruch HP, Windhövel U. Vascular endothelial growth factor (VEGF) a valuable serum tumour marker in patients with colorectal cancer. Eur J Sur Oncol 2001; 27: 34-42.

26. Gasińska A, Urbański K, Adamczyk A. Prognostic signifcance of in tratumour microvessel density and haemoglobin level in carcinoma of the uterine cervix. Acta Oncol 2002; 41: 437-43.

27. Li X, Liu B, Xiao J, Yuan Y, Ma J, Zhang Y. Roles of VEGF-C and Smad4 in the lymphangiogenesis, lymphatic metastasis, and prognosis in colon cancer. J Gastrointest Surg 2011; 7: 23
28. Osinsky S, Bubnovskaya L, Ganusevich I, Kovelskaya A, Gumenyuk L, Olijnichenko G, Merentsev S. Hypoxia, tumour-associated macrophages, microvessel density, VEGF and matrix metalloproteinases in human gastric cancer: interaction and impact on survival. Clin Transl Oncol 2011; 13: 133-8.

29. Fujimoto K, Hosotani R, Wada M, et al. Expression of two angiogenic factors, vascular endothelial growth factor and platelet- derived endothelial cell growth facto in human pancreatic cancer, and its relationship to angiogenesis. Eur J Cancer 1998; 9: 1439-47.

30. Duff SE, Saunders M, McCredie V, Kumar S, O'Dwyer ST, Jayson GC. Pre-operative plasma levels of vascular endothelial growth factor A, C and D in patients with colorectal cancer. Clin Oncol 2005; 17: 367-71.

31. Vermeulen PB, Gasparini G, Fox SB, et al. Quantification of angiogenesis solid human tumours an: international consensus on the methodology and criteria of evaluation. Eur J Cancer 1996; 14: 2474-84.

32. Biedka M, Roman Makarewicz R, Marszałek A, Sir J. Ocena mikrounaczynienia w raku szyjki macicy - wady i zalety. Onkol Info 2010; 7: 84-92.

33. Dunst J, Kuhnt T, Strauss HG, Krause U, Pelz T, Koelbl H, Haensgen G. Anemia in cervical cancer: Impact on survival, patterns of relapse and association with hypoxia and angiogenesis. Int J Radiat Oncol Biol Phys 2003; 56: 778-87.

34. Obermair A, Wasicky R, Kaider A, Preyer O, Lösch A, Leodolter S, Kölbl H. Prognostic significance of tumor angiogenesis in epithelial ovarian cancer. Cancer Lett 1999; 138: 175-82.

35. Haensgen G, Pigorsch S, Strauss H, Dunst J. Plasma levels of vascular endothelial growth factor (VEGF) in patients with cervical cancers: prognostic significance and impact of platelet count and hemoglobin level. Eur J Cancer 2011 [abstract].

36. Hernandez E, Donohue KA, Anderson LL, Heller PB, Stehman FB. The significance of thrombocytosis in patients with locally advanced cervical carcinoma: a Gynecologic Oncology Group study. Gynecol Oncol 2000; 78: 137-42.

37. Ayhan A, Bozdag G, Taskiran C, Gultekin M, Yuce K, Kucukali T. The value of preoperative platelet count in the prediction of cervical involvement and poor prognostic variables in patients with endometrial carcinoma. Gynecol Oncol 2006; 103: 902-5.

\section{Address for correspondence}

\section{Marta Biedka PhD}

Oddział Radioterapii I

Centrum Onkologii im. F. Łukaszczyka

ul. I. Romanowskiej 2

85-796 Bydgoszcz

tel. +48523743374

e-mail: martabiedka@tlen.pl

Submitted: $\quad 20.06 .2010$

Accepted: $\quad 25.07 .2011$ 